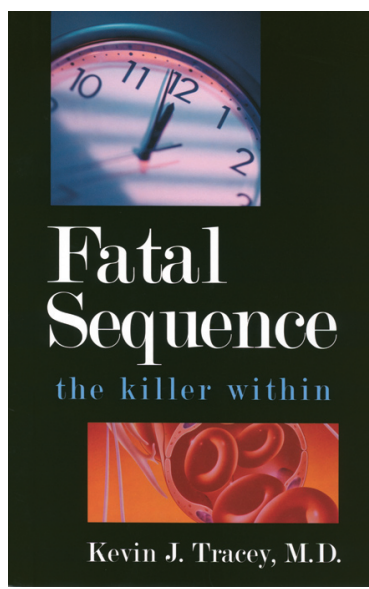

\section{Fatal sequence}

\section{The killer within}

Kevin J. Tracey

Dana Press. New York, New York, USA. 2005.

225 pp. \$23.95. ISBN: 1-932-59406-X (hardcover).

\section{Reviewed by Peter A. Ward}

University of Michigan Medical School, Ann Arbor, Michigan, USA.

E-mail: pward@umich.edu
। n Fatal sequence: the killer within, Kevin Tracey takes the reader on a long journey that begins with his training in general surgery at New York Hospital/Cornell Medical Center. During Tracey's rotation through New York Hospital's renowned burn unit as a second-year surgical resident, he came into contact with a desperately ill 11-month-old girl, Janice, who was not expected to survive after extensive scald burns. Tracey describes in great detail the way in which extensive burn injury causes a cataclysmic hypermetabolic state that threatens to cause death by exhausting all available sources of energy in the body, the loss of body fluids after burn injury together with the imperative need to replace these lost fluids and proteins, and the imminent threat of lethal bacterial infection. These changes and how they are dealt with represent the underpinnings of modern care for burn patients. Tracey's description of how families and relatives as well as physicians, nurses, and staff cope with the extraordinary stresses of the burn unit explains how these individuals are able to deal with what ultimately seems to be a hopeless and overwhelming situation. It is fascinating to hear of how families of patients provide emotional support and encouragement to caregivers of burn patients when the caregivers are about to abandon any hope for recovery of the patient. It is not often we hear such thoughts expressed by physicians directly involved in patient care. We also get unusual snippets of how surgeons realign their psyches in the operating room in order to cope with attendant stresses. We are made aware of the tremendous frustration of physi- cians who are unable to really understand the pathophysiology of severe sepsis and septic shock. At times, this reduces these caregivers to providing supportive therapy and to an extent functioning as bystanders who can neither anticipate nor predict the clinical course in a septic patient, which in the setting of burns can change rapidly and profoundly.

Fatal sequence is not a Lewis Thomas type of story that holds wide appeal to the general public by presenting a positive, philosophical overview. Janice expires unexpectedly with cardiac standstill only days before planned discharge. We hear of Tracey's enormous feelings of guilt and frustration, his decision not to attend Janice's funeral or ever again communicate with her family, and his recurring nightmares after her death. In fact, he interrupts his clinical training to pursue a two-year research study, trying to understand more about the pathophysiology of sepsis. By his own admission, his ultimate pursuit of neurosurgery seems related at least in part to the loss of his mother from glioblastoma multiforme when he was a very young child. The outcome of his retreat into the research laboratory reinforces in Tracey the conviction that bacterial endotoxin (lipopolysaccharide, LPS), TNF- $\alpha$, and HMGB-1 are important mediators of septic shock and that production of many of these mediators is controlled by vagal tone and engagement of the acetylcholine $\alpha 7$ receptor. His strongly held convictions about the role of LPS, TNF- $\alpha$, and perhaps HMGB-1 in sepsis flies in the face of several clinical trials in which anti-TNF- $\alpha$ and soluble TNF- $\alpha$ RI have failed to show efficacy in sepsis. Tracey suggests that the failure of clinical trials in sepsis was due to improper design of the trials. Do LPS and TNF- $\alpha$ play a prima facie role in sepsis? Perhaps. Perhaps not. This is still a highly contestable proposition.

None of this detracts from Tracey's very analytical overview of sepsis, his riveting description of the burn unit, testimonials from adults who have lived to tell what it is like to personally experience sepsis, and his evidence of how the central nervous system regulates production of cytokine and chemokines and how this safety valve may be impaired in sepsis, opening the floodgates for dangerous levels of powerful inflammatory mediators. Tracey has played a key role in advancing the concept of the brain regulating the inflammatory cascade in sepsis. As a clinician and a highly regarded scientist, Tracey has done a beautiful job in helping us to understand the tremendous challenges of sepsis and the toll this condition exacts on patients, their families, and caregivers. It is also apparent that we are woefully ignorant of the details of how and why sepsis develops and how to predict the clinical course of sepsis in any given patient. This is true in spite of the fact that sepsis as a killer is third only to cardiovascular disease and cancers. Because of its technical and scientific detail, Fatal sequence will likely find its readership chiefly among the scientific community, but in some ways, it seems to fill an existing hole in the lay literature, which includes many stories of cancer survivors but few on survivors of sepsis. In general, Fatal sequence is an important contribution that underscores our lack of understanding about sepsis and therefore the difficulty in designing more effective therapy. 